\title{
The Five-Loop Beta-Function of QCD
}

\author{
J.H. Kühn* \\ Institut für Theoretische Teilchenphysik, Karlsruher Institut für Technologie (KIT), \\ 76128 Karlsruhe, Germany \\ E-mail: johann.kuehn@kit.edu
}

Recent developments in perturbative QCD, leading to the beta function in five-loop approximation, are presented. In a first step the two most important decay modes of the Higgs boson are discussed: decays into a pair of gluons and, alternatively, decays into a bottom-antibottom quark pair. Subsequently the $\alpha_{s}^{4}$ corrections to the vector- and axial-vector correlator are discussed. These are the essential ingredients for the evaluation of the QCD corrections to the cross section for electron-positron annihilation into hadrons at low and at high energies, to the hadronic decay rate of the $\tau$ lepton and for the $Z$-boson decay rate into hadrons. Finally we present the prediction for the QCD beta-function in five-loop approximation, discuss the analytic structure of the result and compare with experiment at low and at high energies.

PACS: $12.38 .-t \quad 12.38 . \mathrm{Bx}$

13th International Symposium on Radiative Corrections (Applications of Quantum Field Theory to Phenomenology)

25-29 September, 2017

St. Gilden, Austria

${ }^{*}$ Speaker. 


\section{Introduction}

During the past years significant progress has been made in the evaluation of higher order QCD corrections to inclusive decay rates. In particular the evaluation of QCD corrections to scalarand vector-current correlators has been pushed to $\mathscr{O}\left(\alpha_{s}^{4}\right)[1,2,3]$. Also, along the same line, the evaluation of the QCD beta function has been performed in fifth order [4] and indeed also this result has been confirmed (and extended to a generic gauge group) by three new, independent calculations $[5,6,7]$.

\section{Dominant Higgs boson decay modes}

The two most important decay modes of the Higgs boson are the top quark mediated decay channel into two gluons and the decay into a bottom plus antibottom quark. The higher order corrections to these modes have been evaluated up to $\mathscr{O}\left(\alpha_{s}^{5}\right)[8,9]$ for the two-gluon channel (very recently even up to $\mathscr{O}\left(\alpha_{s}^{6}\right)$ [3]) and up to $\mathscr{O}\left(\alpha_{s}^{4}\right)$ for the $b \bar{b}$ channel [1,3]. Mixed terms related to the $g g$ and the $b \bar{b}$ mode are treated in [10]. These two modes constitute the dominant Higgs decay channels with branching ratios around $15 \%$ for the two-gluon and close to $60 \%$ for the $b \bar{b}$ mode.

In total one finds for the two-gluon channel

$$
\Gamma(H \rightarrow g g)=\Gamma_{\text {Born }}(H \rightarrow g g) \times K
$$

with

$$
\begin{gathered}
\Gamma_{\mathrm{Born}}(H \rightarrow g g)=\frac{G_{F} M_{H}^{3}}{36 \pi \sqrt{2}}\left(\frac{\alpha_{s}^{\left(n_{l}\right)}\left(M_{H}\right)}{\pi}\right)^{2}, \\
K=1+17.9167 a_{s}^{\prime}+152.5\left(a_{s}^{\prime}\right)^{2}+381.5\left(a_{s}^{\prime}\right)^{3} .
\end{gathered}
$$

Here $a_{s}^{\prime}=\alpha_{s} / \pi$. It is quite remarkable that the residual scale dependence is reduced quite drastically, from $\pm 24 \%$ in LO to $\pm 22 \%$ in NLO down to $\pm 10 \%$ in $N^{2} L O$ and $\pm 3 \%$ in $\mathrm{N}^{3} \mathrm{LO}$.

The second, and in fact dominant dominant decay mode of the Higgs boson is the $b \bar{b}$ channel. The decay rate into a quark-antiquark pair, generically denoted by $f \bar{f}$, is given by

$$
\Gamma(H \rightarrow \bar{f} f)=\frac{G_{F} M_{H}}{4 \sqrt{2} \pi} m_{f}^{2} \widetilde{R}\left(s=M_{H}^{2}\right)
$$

where $\tilde{R}(s)=\operatorname{Im} \tilde{\Pi}(-s-i \varepsilon) /(2 \pi s)$ stands for the absorptive part of the scalar two-point correlator

$$
\widetilde{\Pi}\left(Q^{2}\right)=(4 \pi)^{2} i \int d x e^{i q x}\left\langle 0\left|T\left[J_{f}^{\mathrm{S}}(x) J_{f}^{\mathrm{S}}(0)\right]\right| 0\right\rangle .
$$

In five-loop approximation the absorptive part has been obtained in [1] and recently confirmed in $[3,6,7]$.

For $n_{f}=5$, the physically relevant result, it is given by

$$
\begin{aligned}
\tilde{R}\left(s=M_{H}^{2}, \mu=M_{H}\right) & =1+5.667 a_{s}+29.147 a_{s}^{2}+41.758 a_{s}^{3}-825.7 a_{s}^{4} \\
& =1+0.2041+0.0379+0.0020-0.00140
\end{aligned}
$$


In the last equation we have substituted $a_{s}\left(m_{H}\right)=\alpha_{s} / \pi=0.0360$, valid for a Higgs mass of $125 \mathrm{GeV}$ and $\alpha_{s}\left(M_{Z}\right)=0.118$. The significant compensation between $\mathscr{O}\left(\alpha_{s}^{3}\right)$ and $\mathscr{O}\left(\alpha_{s}^{4}\right)$ term may be interpreted as a consequence of an accidentally small coefficient of the $\alpha_{s}^{3}$ term.

In total this leads to a dominant contribution of the $b \bar{b}$ mode with a branching ratio close to $60 \%$. Note that an important ingredient in this context is the mass of the bottom quark at the scale of $m_{H}$, which has been taken as [13]

$$
m_{b}\left(m_{H}\right)=2771 \pm\left. 8\right|_{m_{b}} \pm\left. 15\right|_{\alpha_{s}} \mathrm{MeV} .
$$

In fact it is well known that quark masses are conveniently defined to depend on a renormalization scale

$$
\left.\mu^{2} \frac{d}{d \mu^{2}} m\right|_{g^{0}, m^{0}}=m \gamma_{m}\left(a_{s}\right) \equiv-m \sum_{i \geq 0} \gamma_{i} a_{s}^{i+1}
$$

with $a_{s}=\alpha_{s} / \pi$. The coefficients $\gamma_{i}$ of the quark mass anomalous dimension $\gamma_{m}$ are known from $\gamma_{0}$ to $\gamma_{4}$ and thus in five-loop order [14]. In numerical form and for $S U(3)$ it is given by

$$
\begin{aligned}
& \gamma_{m}= \\
- & a_{s}-a_{s}^{2}\left(4.20833-0.138889 n_{f}\right) \\
- & a_{s}^{3}\left(19.5156-2.28412 n_{f}-0.0270062 n_{f}^{2}\right) \\
- & a_{s}^{4}\left(98.9434-19.1075 n_{f}+0.276163 n_{f}^{2}+0.00579322 n_{f}^{3}\right) \\
- & a_{s}^{5}\left(559.7069-143.6864 n_{f}+7.4824 n_{f}^{2}+0.1083 n_{f}^{3}-0.000085359 n_{f}^{4}\right) .
\end{aligned}
$$

Let us note in passing that quite recently also the result for a general gauge group has been obtained $[15,16]$.

\section{3. $Z$ decay in $\mathscr{O}\left(\alpha_{s}^{4}\right)$}

In view of asymptotic freedom, perturbative QCD can be applied at vastly different energy scales, despite the dramatic variation of the strong coupling between the mass of the $\tau$ lepton and, for example, the mass of the $Z$ boson. Starting, for example, at the scale of the $\tau$-lepton with

$$
\alpha_{s}\left(m_{\tau}\right)=0.332 \pm\left. 0.005\right|_{\exp } \pm\left. 0.015\right|_{t h}
$$

four loop running and matching at the flavour thresholds leads to the reduction of the strong coupling at the scale of the $Z$ boson mass [2]

$$
\alpha_{s}\left(M_{Z}\right)=0.1202 \pm\left. 0.006\right|_{\text {exp }} \pm\left. 0.0018\right|_{\text {th }} \pm\left. 0.0003\right|_{\text {evol }}
$$

by a factor three and a reduction of the uncertainties by nearly a factor ten. The final result is in remarkable agreement with the direct determination of $\alpha_{s}$ from $Z$ decays which leads to $\alpha_{s}=$ $0.1190 \pm\left. 0.0026\right|_{\exp }$ and a small theory error. Note that the dominant term in the QCD corrections for $Z$ decays is identical to the correction term for $\tau$ decays. However, starting from $\mathscr{O}\left(\alpha_{s}^{2}\right)$, one receives additional, new terms in the $Z$ boson case. These arise from so called singlet contributions which in turn are different for the vector and the axial vector part. 
In total one finds for the QCD corrected decay rate of the $Z$ boson (neglecting for the moment mass suppressed terms of $\mathrm{O}\left(m_{b}^{2} / M_{Z}^{2}\right)$ and electroweak corrections)

$$
R^{\mathrm{nc}}=3\left[\sum_{f} v_{f}^{2} r_{\mathrm{NS}}^{V}+\left(\sum_{f} v_{f}\right)^{2} r_{\mathrm{S}}^{V}+\sum_{f} a_{f}^{2} r_{\mathrm{NS}}^{A}+r_{\mathrm{S} ; \mathrm{t}, \mathrm{b}}^{A}\right]
$$

The relative importance of the different terms is best seen from the results of the various $r$ ratios introduced above. In numerical form [17]

$$
\begin{aligned}
r_{\mathrm{NS}}= & 1+a_{s}+1.4092 a_{s}^{2}-12.7671 a_{s}^{3}-79.9806 a_{s}^{4} \\
r_{\mathrm{S}}^{V}= & -0.4132 a_{s}^{3}-4.9841 a_{s}^{4}, \\
r_{\mathrm{S}: \mathrm{t}, \mathrm{b}}^{A}= & \left(-3.0833+l_{t}\right) a_{s}^{2}+\left(-15.9877+3.7222 l_{t}+1.9167 l_{t}^{2}\right) a_{s}^{3} \\
& +\left(49.0309-17.6637 l_{t}+14.6597 l_{t}^{2}+3.6736 l_{t}^{3}\right) a_{s}^{4},
\end{aligned}
$$

with $a_{s}=\alpha_{s}\left(M_{Z}\right) / \pi$ and $l_{t}=\ln \left(M_{Z}^{2} / M_{t}^{2}\right)$. Using for the pole mass $M_{t}$ the value $172 \mathrm{GeV}$, the axial singlet contribution in numerical form is given by

$$
r_{\mathrm{S} ; \mathrm{t}, \mathrm{b}}^{A}=-4.3524 a_{s}^{2}-17.6245 a_{s}^{3}+87.5520 a_{s}^{4} .
$$

Let us recall the basic aspects of these results:

- The non-singlet term dominates all different channels. It starts in Born approximation and is identical for $\tau$ decay, for $\sigma\left(e^{+} e^{-} \rightarrow\right.$ hadrons) through the vector current (virtual photon) and for $\Gamma(Z \rightarrow$ hadrons $)$ through vector and axial current.

- The singlet axial term starts in order $\alpha_{s}^{2}$, is present in $Z \rightarrow$ hadrons and depends on $\ln M_{Z}^{2} / M_{t}^{2}$. Its origin is the strong imbalance between the masses of the top and the bottom quark [18].

- The singlet vector term is present both in $\gamma^{*} \rightarrow$ hadrons and $Z \rightarrow$ hadrons and starts in $\mathscr{O}\left(\alpha_{s}^{3}\right)$.

- All three terms are known up to order $\alpha_{s}^{4}$ and the total rate is remarkably stable under scale variations.

\section{Five-loop $\beta$ function}

Asymptotic freedom, manifest by a decreasing coupling with increasing energy, can be considered as the basic prediction of nonabelian gauge theories and was crucial for establishing QCD as the theory of strong interactions. The dominant, leading order prediction $[19,20]$ was quickly followed by the corresponding two- $[21,22]$ and three-loop [23, 24] results. Subsequently it took more than 15 years until the four-loop result was evaluated [25] and another seven years until this result was confirmed by an independent calculation [26]. Now, finally, the five-loop result for QCD became available [4], quickly confirmed and generalized to an arbitrary gauge group [5, 6, 7].

There are several reasons to push the QCD $\beta$-function to an order as high as possible. From the practical side it is important to compare experiment and theory prediction with the best achievable precision. From the theoretical side one expects that the perturbative series at some point starts 
to demonstrate its asymptotic divergence, shown by significantly increasing terms. However, as shown below, even up to fifth order the series exhibits a remarkably smooth behaviour with continuously decreasing perturbative coefficients. Let us, in a first step, recall the coefficients of the QCD $\beta$-function defined by

$$
\beta\left(a_{s}\right)=\mu^{2} \frac{d}{d \mu^{2}} a_{s}(\mu)=-\sum_{i \geq 0} \beta_{i} a_{s}^{i+2} .
$$

Using the same tools as those discussed in $[2,14]$ the $\beta$-function in fifth order is given by

$$
\begin{aligned}
\beta_{0} & =\frac{1}{4}\left\{11-\frac{2}{3} n_{f},\right\}, \beta_{1}=\frac{1}{4^{2}}\left\{102-\frac{38}{3} n_{f}\right\}, \\
\beta_{2} & =\frac{1}{4^{3}}\left\{\frac{2857}{2}-\frac{5033}{18} n_{f}+\frac{325}{54} n_{f}^{2}\right\}, \\
\beta_{3} & =\frac{1}{4^{4}}\left\{\frac{149753}{6}+3564 \zeta_{3}-\left[\frac{1078361}{162}+\frac{6508}{27} \zeta_{3}\right] n_{f}\right. \\
+ & {\left.\left[\frac{50065}{162}+\frac{6472}{81} \zeta_{3}\right] n_{f}^{2}+\frac{1093}{729} n_{f}^{3}\right\}, } \\
\beta_{4}= & \frac{1}{4^{5}}\left\{\frac{8157455}{16}+\frac{621885}{2} \zeta_{3}-\frac{88209}{2} \zeta_{4}-288090 \zeta_{5}\right. \\
+ & n_{f}\left[-\frac{336460813}{1944}-\frac{4811164}{81} \zeta_{3}+\frac{33935}{6} \zeta_{4}+\frac{1358995}{27} \zeta_{5}\right] \\
+ & n_{f}^{2}\left[\frac{25960913}{1944}+\frac{698531}{81} \zeta_{3}-\frac{10526}{9} \zeta_{4}-\frac{381760}{81} \zeta_{5}\right] \\
+ & n_{f}^{3}\left[-\frac{630559}{5832}-\frac{48722}{243} \zeta_{3}+\frac{1618}{27} \zeta_{4}+\frac{460}{9} \zeta_{5}\right] \\
+ & \left.n_{f}^{4}\left[\frac{1205}{2916}-\frac{152}{81} \zeta_{3}\right]\right\} .
\end{aligned}
$$

This result has, in the meantime, been confirmed in $[5,6,7]$ and even extended to an arbitrary, simple, compact Lie group. The surprising pattern of the delayed appearance of higher transcendentals, already observed in lower orders, repeats itself in the present case: The transcendental numbers $\zeta_{6}$ and $\zeta_{7}$ that could be present in $\beta_{4}$ in principle, are evidently absent, similarly to the absence of $\zeta_{4}$ and $\zeta_{5}$ in the result for $\beta_{3}$.

As stated before, the smallness of the higher order coefficients, in particular for the $n_{f}$-values of interest, leads to a remarkable stabilization of the results. The excellent agreement between $\alpha_{s}$ values from vastly different energy scales indeed persists in higher orders. Let us, as a typical example, recall the comparison between the strong coupling at the scales of $m_{\tau}$ and $M_{Z}$. Starting with the value $\alpha_{s}\left(m_{\tau}\right)=0.33 \pm 0.014$ one arrives, after running and matching at the charm and bottom threshold at the value $\alpha_{s}^{(5)}=0.1198 \pm 0.0015$. From the direct measurement of $Z$-boson decays combined in the electroweak precision data, on the other hand, one obtains the result $\alpha_{s}^{(5)}=$ $0.1197 \pm 0.0028$ in remarkable agreement with the previous value. 


\section{Summary}

A sizable number of four- and five-loop QCD results has been evaluated during the past years. $\mathscr{O}\left(\alpha_{s}^{4}\right)$ corrections of Higgs boson decays to fermions, of $\tau$-lepton decays to hadrons, $Z$ decays to hadrons and of corrections to the familiar $R$ ratio (with $R \equiv \sigma\left(e^{+} e^{-} \rightarrow\right.$ hadrons) $/ \sigma\left(e^{+} e^{-} \rightarrow\right.$ $\left.\mu^{+} \mu^{-}\right)$) are among the most prominent examples. These calculations have been complemented by the most recent result along the same lines, the five-loop QCD $\beta$ function. No sign of an onset of the asymptotically expected divergence of the series is observed. Excellent agreement between theory and experiment for a large number of predictions is observed. For the moment the precision of the theoretical prediction is significantly ahead of the experimental results.

\section{Acknowledgements}

The author would like to thank P. Baikov and K. Chetyrkin for a pleasant collaboration in numerous topics discussed in this paper.

\section{References}

[1] P. A. Baikov, K. G. Chetyrkin and J. H. Kühn, Scalar correlator at $\mathscr{O}\left(\alpha_{s}^{4}\right)$, Higgs decay into b-quarks and bounds on the light quark masses, Phys. Rev. Lett. 96 (2006) 012003, [hep-ph / 0511063 ].

[2] P. A. Baikov, K. G. Chetyrkin and J. H. Kühn, Order $\alpha_{s}(s)^{4} Q C D$ Corrections to Z and tau Decays, Phys. Rev. Lett. 101 (2008) 012002, [0801.1821].

[3] F. Herzog, B. Ruijl, T. Ueda, J. A. M. Vermaseren and A. Vogt, On Higgs decays to hadrons and the $R$-ratio at $N^{4} L O, J H E P 08$ (2017) 113, [1707.01044].

[4] P. A. Baikov, K. G. Chetyrkin and J. H. Kühn, Five-Loop Running of the QCD coupling constant, Phys. Rev. Lett. 118 (2017) 082002, [1606.08659].

[5] F. Herzog, B. Ruijl, T. Ueda, J. A. M. Vermaseren and A. Vogt, The five-loop beta function of Yang-Mills theory with fermions, JHEP 02 (2017) 090, [1701.014 0 4].

[6] T. Luthe, A. Maier, P. Marquard and Y. Schroder, The five-loop Beta function for a general gauge group and anomalous dimensions beyond Feynman gauge, 1709.07718.

[7] K. G. Chetyrkin, G. Falcioni, F. Herzog and J. A. M. Vermaseren, Five-loop renormalisation of QCD in covariant gauges, 1709.08541 .

[8] P. A. Baikov and K. G. Chetyrkin, Top Quark Mediated Higgs Boson Decay into Hadrons to Order $\alpha_{s}^{5}$, Phys. Rev. Lett. 97 (2006) 061803, [hep-ph/ 0604194$]$.

[9] J. Davies, M. Steinhauser and D. Wellmann, Hadronic Higgs boson decay at order $\alpha_{s}^{4}$ and $\alpha_{s}^{5}$, in 25th International Workshop on Deep Inelastic Scattering and Related Topics (DIS 2017) Birmingham, UK, April 3-7, 2017, 2017. 1706.00624.

[10] J. Davies, M. Steinhauser and D. Wellmann, Completing the hadronic Higgs boson decay at order $\alpha_{s}^{4}$, Nucl. Phys. B920 (2017) 20-31, [1703.02988].

[11] K. G. Chetyrkin, B. A. Kniehl and M. Steinhauser, Hadronic Higgs decay to order $\alpha_{s}^{4}$, Phys. Rev. Lett. 79 (1997) 353-356, [hep-ph/9705240]. 
[12] K. G. Chetyrkin, B. A. Kniehl and M. Steinhauser, Decoupling relations to $\mathscr{O}\left(\alpha_{s}^{3}\right)$ and their connection to low-energy theorems, Nucl. Phys. B510 (1998) 61-87, [hep-ph/9708255].

[13] F. Herren and M. Steinhauser, Version 3 of RunDec and CRunDec, 1703.03751.

[14] P. A. Baikov, K. G. Chetyrkin and J. H. Kühn, Quark Mass and Field Anomalous Dimensions to $\mathscr{O}\left(\alpha_{s}^{5}\right)$, JHEP 10 (2014) 076, [1402.6611].

[15] T. Luthe, A. Maier, P. Marquard and Y. Schröder, Five-loop quark mass and field anomalous dimensions for a general gauge group, JHEP 01 (2017) 081, [1612 . 05512].

[16] P. A. Baikov, K. G. Chetyrkin and J. H. Kühn, Five-loop fermion anomalous dimension for a general gauge group from four-loop massless propagators, JHEP 04 (2017) 119, [1702 . 01458 ].

[17] P. A. Baikov, K. G. Chetyrkin, J. H. Kühn and J. Rittinger, Complete $\mathscr{O}\left(\alpha_{s}^{4}\right)$ QCD Corrections to Hadronic Z-Decays, Phys. Rev. Lett. 108 (2012) 222003, [1201 . 5804 ].

[18] B. A. Kniehl and J. H. Kühn, QCD Corrections to the Axial Part of the Z Decay Rate, Phys. Lett. B224 (1989) 229.

[19] D. J. Gross and F. Wilczek, Ultraviolet behavior of non-abelian gauge theories, Phys. Rev. Lett. 30 (1973) 1343-1346.

[20] H. D. Politzer, Reliable perturbative results for strong interactions?, Phys. Rev. Lett. 30 (1973) 1346-1349.

[21] W. E. Caswell, Asymptotic behavior of nonabelian gauge theories to two loop order, Phys. Rev. Lett. 33 (1974) 244.

[22] D. R. T. Jones, Two loop diagrams in yang-mills theory, Nucl. Phys. B75 (1974) 531.

[23] O. V. Tarasov, A. A. Vladimirov and A. Y. Zharkov, The gell-mann-low function of QCD in the three loop approximation, Phys. Lett. $\mathbf{B 9 3}$ (1980) 429-432.

[24] S. A. Larin and J. A. M. Vermaseren, The three loop QCD beta function and anomalous dimensions, Phys. Lett. B303 (1993) 334-336, [hep-ph/9302208].

[25] T. van Ritbergen, J. A. M. Vermaseren and S. A. Larin, The four-loop beta function in quantum chromodynamics, Phys. Lett. B400 (1997) 379-384, [hep-ph / 9701390$].$

[26] M. Czakon, The Four-loop QCD beta-function and anomalous dimensions, Nucl.Phys. B710 (2005) 485-498, [hep-ph/0411261]. 